\title{
Conversión de tuberculosis latente en un grupo de pacientes tratados con terapia biológica en una unidad de Reumatología
}

\author{
Conversion of latent tuberculosis in a group of patients \\ treated with biologic therapy in a Rheumatology unit
}

OScAR MAURICIO MUÑoz V., MD., MSc.(1); MAITE HuRTAdo URIARTE, MD. (2); DANIEL FERnÁNDEZ Ávila, MD., MSc (c) $)^{(3)}$; PATRICIA HIDALgo., MD., MSc. ${ }^{(4)}$; JUAN MARTíN GUTIÉRREZ DÁVILA, MD. ${ }^{(5)}$

\section{Resumen}

OBJETIVO: los pacientes que padecen enfermedades reumáticas, en tratamiento con terapia biológica, tienen como consecuencia la inmunosupresión y el riesgo de reactivación de enfermedades latentes crónicas como la tuberculosis. Dado que no existen estimativos de este riesgo en Colombia, se consideró importante realizar un estudio que evaluara la incidencia de tuberculosis latente en esta población.

MÉTODOS: se hizo un estudio descriptivo basado en una cohorte histórica, que incluyó pacientes de la Unidad de Reumatología del Hospital Universitario de San Ignacio, en Bogotá (Colombia). Se evaluaron 78 candidatos a terapia biológica, libres de tuberculosis activa o latente, al ingreso a la cohorte. Se realizó prueba cutánea de tuberculina (Tubersol ${ }^{\circledR}$ Sanofi Pasteur) al inicio de la terapia biológica y controles a los seis meses. Se definió conversión de tuberculosis latente como viraje de PPD mayor a $5 \mathrm{~mm}$ respecto a la inicial.

RESULTADOS: 78 pacientes cumplieron criterios de inclusión. 27 presentaron datos incompletos y por esto fueron excluidos. Se incluyeron para el análisis 51 pacientes, 12 de los cuales presentaron viraje de PPD (23,5\%).

CONCLUSIONES: este estudio demuestra la alta incidencia de conversión de tuberculosis latente y por tanto el alto riesgo al que se someten los pacientes en terapia biológica, punto de partida para optimizar el estudio y seguimiento de los mismos.

Palabras clave: tuberculosis latente, agentes biológicos/efectos adversos, infección/diagnóstico.

\begin{abstract}
OBJECTIVE: Patients with rheumatic diseases who undergo treatment with biologic therapies have immunosuppression as a consequence, and are at risk for reactivation of chronic latent diseases such as tuberculosis. Since there are no estimations of this risk in Colombia, it was considered important to carry out a study aimed at assessing the incidence of latent tuberculosis in this population.

METHODS: A descriptive study based on a historical cohort was carried out. It included patients of the Rheumatology Unit of Hospital Universitario de San Ignacio, in Bogotá (Colombia). 78 candidates for biologic therapy were assessed, who were free of latent or active tuberculosis on admission to the cohort. Cutaneous tuberculin testing (Tubersol ${ }^{\circledR}$ Sanofi Pasteur) was done at the beginning of biologic therapy and controlled after six months. Conversion of latent tuberculosis was defined as a PPD shift greater than $5 \mathrm{~mm}$ with respect to the initial response.
\end{abstract}

RESULTS: 78 patients met inclusion criteria. 25 had incomplete data and were therefore excluded. 51 patients were included for analysis, 12 of whom showed PPD shift (23,5\%).

CONCLUSIONS: This study demonstrates the high incidence of conversion of latent tuberculosis, and therefore the high risk of patients on biologic therapy. This should be a starting point for optimizing the study and followup of these patients.

Keywords: latent tuberculosis, biologic agents/adverse effects, infection/diagnosis.

\footnotetext{
(1)Profesor Asistente de Medicina Interna, Departamento de Medicina Interna. Pontificia Universidad Javeriana. Hospital Universitario San Ignacio.

${ }^{(2)}$ Médico Internista Departamento de Medicina Interna. Pontificia Universidad Javeriana. Hospital Universitario San Ignacio. ${ }^{(3)}$ Médico Internista y Reumatólogo. Profesor Unidad de Reumatología. Departamento de Medicina Interna. Pontificia Universidad Javeriana. Hospital Universitario San Ignacio.

(4) Médico Internista y Neumólogo, Profesor Unidad de Neumología. Departamento de Medicina Interna. Pontificia Universidad Javeriana. Hospital Universitario San Ignacio. ${ }^{(5)}$ Médico Internista y Reumatólogo. Profesor Titular Unidad de Reumatología. Departamento de Medicina Interna. Pontificia Universidad Javeriana. Hospital Universitario San Ignacio.

Correspondencia: Oscar Mauricio Muñoz, Correo electrónico: o.munoz@javeriana. edu.co

Recibido: 11/05/14. Aceptado: 10/06/14.
} 


\section{Introducción}

La tuberculosis (TB) continúa siendo un problema de salud mundial. Se estima que 2 billones de personas están infectadas en el mundo, y que genera 1.5 a 2 millones de muertes al año, convirtiéndose así en la causa de muerte infecciosa número uno (1-3).

Se han realizado grandes esfuerzos para tratar de identificar y contener los casos de tuberculosis, declarada emergencia global por la Organización Mundial de la Salud (OMS) en 1993 (1); sin embargo, continúan las altas tasas de pacientes infectados o portadores (TB latente) perpetuando el inmenso problema de salud mundial.

En Colombia se cuenta con una incidencia de 24 por 100.000 habitantes, y se reportan anualmente más de 11.000 casos nuevos, con una mortalidad de 2,5 por 100.000 personas (4).

Se estima que las personas con TB latente sin defectos inmunológicos tienen un riesgo de $0,1 \%$ de desarrollar TB activa (2), porcentaje que aumenta notablemente en pacientes con alteraciones inmunológicas, de ahí que se recomiende la búsqueda activa de TB latente en pacientes inmunosuprimidos. El manejo con isoniazida reduce el riesgo de desarrollar TB en estos pacientes, que se han identificado por respuesta inmune específica.

Como producto del mejor conocimiento del funcionamiento del sistema inmune y el avance de la biología molecular, en los últimos treinta años se ha presentado un cambio significativo en las opciones terapéuticas de algunas enfermedades reumáticas con el advenimiento de las terapias biológicas, las cuales buscan la modulación molecular implicada en la fisiopatología de la enfermedad autoinmune. Estas nuevas terapias ofrecen oportunidades de tratamiento, control de los síntomas y de la evolución de la enfermedad, así como mejoría en su pronóstico. No obstante, generan riesgo de sufrir enfermedades infecciosas al disminuir la respuesta inmune ante ciertos patógenos como el bacilo de la tuberculosis. Un ejemplo es el factor de necrosis tumoral (FNT- $\alpha$ ), citoquina de gran importancia en el funcionamiento del sistema inmune, aumentando la actividad fagocí- tica de los macrógafos y la muerte intracelular de los microorganismos por la vía de las caspasas mediado por oxígeno y nitrógeno. El FNT- $\alpha$ promueve la liberación de citoquinas y la formación del granuloma. En ausencia de éste se produce desintegración, se liberan y diseminan bacilos, y se aumenta, por ende, el riesgo de progresión a TB activa (1).

Algunos estudios han mostrado que en los pacientes que venían recibiendo terapia anti FNT- $\alpha$ es mayor el riesgo de desarrollar TB. El estudio español BIOBADASER mostró un riesgo del 20 al 40\% de contraer infecciones con el uso de FNT- $\alpha$ (RR ajustado 1,57; IC del 95\%, 0,92-2,67) (5). En el año 2000 se detectó en los pacientes españoles tratados con anti-TNF- $\alpha$ una alta incidencia de TB activa en comparación con lo esperado en la población general (RR de entre 11 y 20 con relación a las artritis reumatoides no expuestas) (6).

En otro estudio hecho en Canadá en 2006, en una cohorte retrospectiva con 112.300 pacientes con artritis reumatoide, se observó una incidencia de TB de 2,2 por 1.000 pacientes año (IC $95 \% 2,0-2,4$ ), en comparación con 2,6 por 1.000 pacientes año (IC $95 \% 1,9-3,3)$ en quienes recibían anti FNT- $\alpha(7)$.

De otra parte, el estudio británico BSRBR reportó una incidencia de TB del 0,5, 1,5 y 0,9 por 1.000 pacientes/año, respectivamente para etanercept, infliximab y adalimumab, con un promedio de presentación entre dos a nueve meses después del inicio de la terapia biológica (8).

Así mismo, un metanálisis llevado a cabo con la colaboración de Cochrane acerca los efectos adversos de los biológicos en los diferentes grupos, comparó más de 163 estudios con 50.010 pacientes y mostró un aumento del riesgo de reactivación de la TB (OR 4,66, IC 95\% 1,18-18,60; NNTH: 681, IC 95\% 143-14.706) en comparación con el control (9).

La mayoría de casos de TB obedece a la reactivación de TB latente con una progresión más rápida y diseminada. De ahí parte la importancia del diagnóstico oportuno, para recibir tratamiento a tiempo y evitar la progresión, mortalidad y complicaciones que esta implica (10-13).

Revista Colombiana de Neumología Vol 26 No 3 | 2014 
Por ende, se consideró imperativo hacer una búsqueda exhaustiva de pacientes en riesgo, teniendo en cuenta que no se trata de una condición estática, en la que los pacientes bajo terapia inmunomoduladora con medicamentos biológicos están en exposición constante al bacilo y pueden ser infectados por Mycobacterium tuberculosis en cualquier momento de su tratamiento, y están en alto riesgo de evolucionar a enfermedad activa y diseminada. Adicionalmente, se creyó pertinente adelantar esta investigación con el objetivo de verificar la situación actual de un grupo de pacientes reumáticos del Hospital Universitario San Ignacio (HUSI).

\section{Métodos}

Se realizó un estudio observacional, descriptivo, de una cohorte histórica, tomando como población de estudio todos los pacientes que venían en manejo con agentes biológicos y asistían a seguimiento periódico en la unidad de reumatología del HUSI. Para ingresar a la cohorte a los pacientes candidatos a terapia biológica se les debía descartar TB activa o TB latente previa, para lo cual se realizó PPD (prueba de tuberculina) al inicio (se consideró negativo el valor menor a $5 \mathrm{~mm}$ ), y prueba Booster a los 7 a 10 días entre aquellos con reporte negativo (14). Así mismo, se les realizó a todos una radiografía de tórax; de ser normales se consideraban candidatos para ingresar al estudio.

La información respecto a las características basales se tomó directamente de la historia clínica electrónica. Las variables estudiadas fueron: edad, género, estrato socioeconómico, hacinamiento, antecedente de contacto respiratorio, tratamiento biológico actual, tiempo de tratamiento, tratamiento anterior, y uso de otros inmunosupresores. En caso de datos faltantes, se completaron a través de entrevista telefónica.

En todos los casos se registró información de las pruebas de tuberculina tomadas al menos seis meses después del inicio de la terapia biológica. La aplicación de PPD se realizó utilizando la técnica de Mantoux con $0,1 \mathrm{~mL}$ de derivado proteico purificado que contiene 5 TU (Tubersol ${ }^{\circledR}$ Sanofi Pasteur) de aplicación intradérmica en el tercio medio de la superficie anterior del antebrazo derecho. Se realizó la lectura entre 48 y 72 horas después de la aplicación. El resultado se determinó de acuerdo con la medición del diámetro de la induración transverso al eje del antebrazo y fue expresado en milímetros (la aparición de vesículas o necrosis se consideró como indicativa de infección tuberculosa). El personal encargado de la lectura eran personas con entrenamiento quienes rutinariamente realizan esta prueba. Considerando que un alto número de pacientes no asisten para la realización de la prueba de tuberculina de control y buscando disminuir la tasa de datos faltantes en la evaluación del desenlace, se les ofreció a todos los pacientes que no tenían evaluación de conversión de tuberculina, después de seis meses de iniciada la terapia biológica, la realización de la prueba en forma domiciliaria.

Se definió viraje de PPD como aquellos que presentaban en las PPD de control un cambio mayor a 5 $\mathrm{mm}$ respecto a la inicial (15). Se excluyeron para los análisis aquellos pacientes en quienes, a pesar de los esfuerzos realizados, no se pudo realizar la prueba de tuberculina de control.

La recolección de datos y la realización de las pruebas se efectuaron en el período junio de 2012 a junio de 2013. Las pruebas para evaluar la conversión de tuberculina se hicieron con intervalos de tratamiento diferentes, posterior al inicio de la terapia biológica, siendo seis meses el tiempo mínimo aceptado, lo cual constituye una limitante del estudio.

\section{Análisis estadístico}

El análisis de los datos se efectuó a través de estadística descriptiva con medidas de tendencia central para las variables continuas y cálculo de frecuencias para las variables categóricas, mediante el programa estadístico STATA 10. Dado que se trata de un estudio observacional descriptivo, no se hicieron pruebas estadísticas para evaluar asociaciones entre factores de riesgo y viraje de la prueba.

\section{Resultados}

Para el estudio se obtuvo información de 97 pacientes tratados con terapias biológicas en la unidad de Reumatología del Hospital Universitario San 
Ignacio. De ellos, 19 presentaron TB latente, documentada antes del inicio de la terapia.

Hubo 78 pacientes que cumplieron criterios de inclusión; sin embargo, 31 presentaron inicialmente datos incompletos, ya que no tenían disponible la prueba de tuberculina de control. A todos ellos se les ofreció la realización de prueba de tuberculina domiciliaria, procedimiento que aceptaron 4 pacientes. Los 27 pacientes restantes fueron excluidos para el análisis (figura 1).

Las características basales de los 51 pacientes incluidos se muestran en la tabla 1. La mayoría de la población tuvo indicación de terapia biológica por artritis reumatoide (91\%), y los medicamentos más utilizados fueron adalimumab $(31,4 \%)$ y rituximab $(31,4 \%)$.

Doce de 51 incluidos en el análisis (23,5\%), presentaron conversión de TB latente. En la tabla 2 se comparan las características de los pacientes

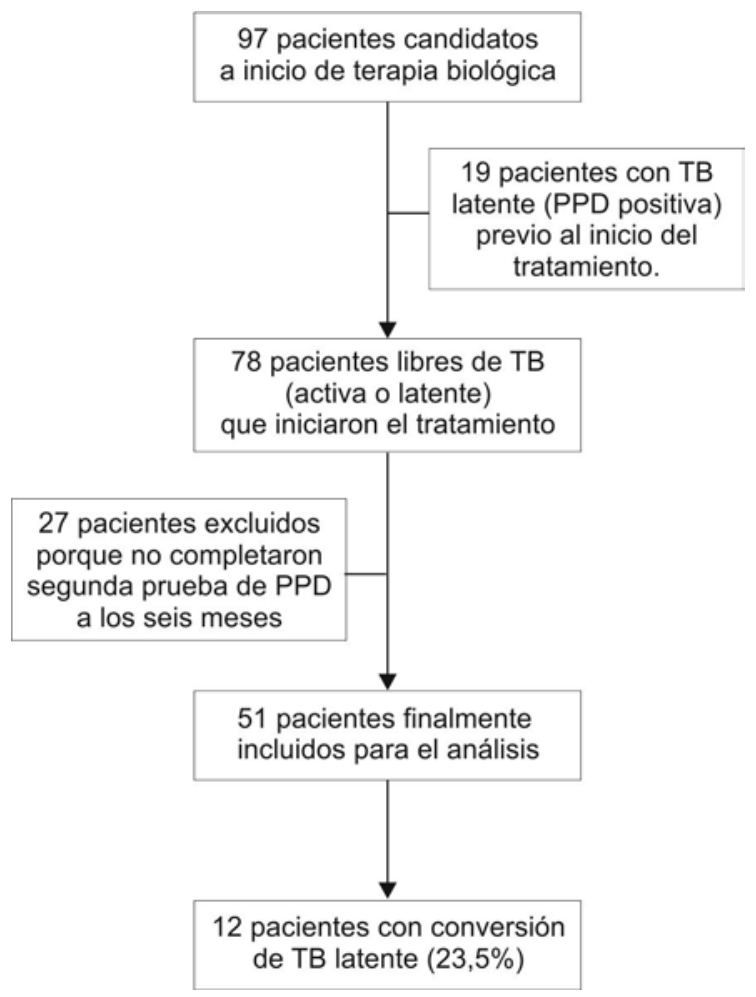

Figura 1. Flujograma de pacientes incluidos y excluidos del análisis. que desarrollaron o no conversión en la prueba de tuberculina.

Respecto a la incidencia de conversión de TB latente de acuerdo con el medicamento utilizado, llamó la atención este fenómeno entre los pacientes tratados con rituximab $(37,5 \%)$, la cual fue superior a la presentada con otros agentes biológicos. No se realizaron pruebas estadísticas para evaluar asociaciones entre los factores de riesgo y los even-

Tabla 1. Características de base de los 51 pacientes incluidos en el análisis.

\begin{tabular}{|c|c|}
\hline Características & Medida de resumen \\
\hline $\begin{array}{l}\text { Edad (años), } \\
\text { Promedio } \pm \text { DE }\end{array}$ & $51,6 \pm 13,15$ \\
\hline Género, n (\%) & $\begin{array}{l}\text { Hombres } 10(19,6) \\
\text { Mujeres } 41(89,39)\end{array}$ \\
\hline $\begin{array}{l}\text { Estrato socioeconómico, } \\
\mathrm{n}(\%)\end{array}$ & $\begin{array}{l}\text { Estrato 1: } 5(9,8) \\
\text { Estrato 2: } 10(19,61) \\
\text { Estrato 3: } 26(50,98) \\
\text { Estrato 4: } 6(11,76) \\
\text { Estrato 5: } 1(1,96) \\
\text { Estrato 6: } 2(3,92)\end{array}$ \\
\hline $\begin{array}{l}\text { Exposición a sintomático } \\
\text { respiratorio, } \mathrm{n}(\%)\end{array}$ & $\begin{array}{l}\text { No presentó: } 38(74,5) \\
\text { Presentó: } 12(23,5) \\
\text { NS/NR } 1(2)\end{array}$ \\
\hline $\begin{array}{l}\text { Uso concomitante de } \\
\text { esteroides, } \mathrm{n}(\%)\end{array}$ & $\begin{array}{l}\text { No tenía: } 26 \text { (51) } \\
\text { Menor de } 15 \mathrm{mg} / \text { día: } 22(43,1) \\
\text { Mayor de } 15 \mathrm{mg} / \text { día: } 3(5,9)\end{array}$ \\
\hline $\begin{array}{l}\text { Diagnóstico de base, } \\
\mathrm{n}(\%)\end{array}$ & $\begin{array}{l}\text { AR: } 42(82,35) \\
\text { Espondilitis anquilosante: } 3(5,8) \\
\text { END: } 2(3,92) \\
\text { EAEI } 1(1,96) \\
\text { LES: } 2(3,92) \\
\text { Síndrome de Sjögren: } 1(1,96)\end{array}$ \\
\hline $\begin{array}{l}\text { Tratamiento Biológico, } \\
\mathrm{n}(\%)\end{array}$ & $\begin{array}{l}\text { Adalimumab: } 16(31,4) . \\
\text { Etanercept: } 5(9,8) . \\
\text { Infliximab: } 6(11,7) . \\
\text { Rituximab: } 16(31,3) \\
\text { Abatacept: } 2(3,92) . \\
\text { Tocilizumab: } 6(11,7) .\end{array}$ \\
\hline Hacinamiento, n (\%) & $\begin{array}{l}\text { No: } 49(96) \\
\text { Si: } 1(2) \\
\text { Sin información: } 1 \text { (2) }\end{array}$ \\
\hline
\end{tabular}

DE: desviación estándar; NS/NR: No sabe/No responde; AR: artritis reumatoide; END: espondiloartropatía no diferenciada; EAEI: espondiloartropatía asociada a enfermedad inflamatoria intestinal; LES: lupus eritematoso sistémico. 
tos de conversión de tuberculina, considerando el diseño observacional descriptivo y el bajo número de eventos.

\section{Discusión}

De acuerdo con los autores, este es el primer estudio que reporta tasa de conversión de viraje de prueba de tuberculina en pacientes con terapia biológica. Es llamativa la tasa alta de viraje de PPD $(23,5 \%)$. Es una limitante importante del estudio el alto número de pacientes en quienes no fue posible realizar la prueba de tuberculina de control; sin embargo, al hacer un análisis de sensibilidad de los datos, se encontró que si ninguno de ellos hubiese presentado conversión, la incidencia sería igualmente alta, aproximadamente mayor al $15 \%$.

Estos datos sugieren que la probabilidad de viraje de la prueba de tuberculina para pacientes que reciben terapia biológica, es muy superior a la esperada en la población general, la cual ha sido reportada como menor al $0,1 \%$ anual en pacientes no inmunosuprimidos (16), y abre la ventana para realizar nuevos estudios prospectivos que determinen de una forma más contundente el riesgo al que se enfrenta esta población. De documentarse en estos estudios tasas similares a las reportadas en la muestra, sería necesario reevaluar la forma en que se tamiza a los pacientes para infección tuberculosa y la indicación de quimioprofilaxis en ellos.

El estudio tiene importantes limitaciones metodológicas; una de ellas es la forma en que se descartó la presencia de TB latente al inicio. Una prueba de tuberculina negativa no permite descartar de forma contundente el diagnóstico de TB latente dado que los pacientes con enfermedades reumáticas pueden presentar anergia asociada a la enfermedad (17). A pesar de estas limitaciones la prueba de tuberculi-

Tabla 2. Características comparativas de los pacientes que presentaron o no conversión de tuberculina.

\begin{tabular}{|c|c|c|}
\hline Características & Pacientes con viraje $(n=12)$ & Pacientes sin viraje $(n=39)$ \\
\hline $\begin{array}{l}\text { Edad (años), } \\
\text { Promedio } \pm \mathrm{DE}\end{array}$ & $51,6 \pm 13$ & $50,74 \pm 14$ \\
\hline \multirow[t]{2}{*}{ Género, n (\%) } & Hombres $2(16,7)$ & Hombres $8(20,5)$ \\
\hline & Mujeres $10(83,3)$ & Mujeres $31(79,5)$ \\
\hline \multirow[t]{4}{*}{ Estrato socioeconómico, n (\%) } & Estrato & Estrato \\
\hline & Estrato 2: $2(16,67)$ & Estrato 2: $9(23,0)$ \\
\hline & Estrato $3: 8(66,6)$ & Estrato 3: $19(48,0)$ \\
\hline & Estrato 4: $2(16,67)$ & Estrato 4: $5(12,0)$ \\
\hline \multirow[t]{2}{*}{ Exposición a sintomático respiratorio, n (\%) } & No presento: $8(66,67)$ & No Presento: $28(71,0)$ \\
\hline & Presento: $4(33,33)$ & Presento: $10(25,0)$ \\
\hline \multirow[t]{3}{*}{ Uso concomitante de esteroides, n (\%) } & No tenía: $7(58,33)$ & No tenía: $17(43,5)$ \\
\hline & $<15$ mg/dL: $5(41,67)$ & <15 mg/dl: $519(48,72)$ \\
\hline & $>15$ mg/dL: $0(0)$ & $>15$ mg/dl: $3(7,69)$ \\
\hline \multirow[t]{2}{*}{ Diagnóstico de base, n (\%) } & AR: $11(91,67)$ & AR $34(87,1)$ \\
\hline & EAEI: $1(8,33)$ & EAEI $0(0,0)$ \\
\hline \multirow[t]{6}{*}{ Tratamiento biológico, n (\%) } & Adalimumab: 2 & Adalimumab: 14 \\
\hline & Etanercept: 1 & Etanercept: 4 \\
\hline & Infliximab: 1 & Infliximab: 5 \\
\hline & Rituximab: 6 & Rituximab: 10 \\
\hline & Abatacept: 1 & Abatacept: 1 \\
\hline & Tocilizumab: 1 & Tocilizumab: 5 \\
\hline
\end{tabular}

DE: desviación estándar; AR: artritis reumatoide; EAEI: espondiloartropatía asociada a enfermedad inflamatoria intestinal. 
na es la mejor herramienta disponible en Colombia para este objetivo, y hace parte de la práctica diaria. Otras pruebas como la evaluación de niveles de interferón gamma, han tenido serias limitaciones para ser interpretadas en pacientes inmunosuprimidos (18), son costosas y no están disponibles habitualmente en nuestro medio.

De igual forma es llamativo el hecho de que seis pacientes en tratamiento con rituximab $(37,5 \%$ de los pacientes que recibieron el medicamento) presentaran conversión de tuberculina. Este dato, sin embargo, debe interpretarse con cautela, dado el bajo número de eventos, y el que no hubo un grupo de control. No obstante, los resultados encienden las alarmas para llevar a cabo estudios que evalúen de forma cuidadosa el riesgo para cada agente biológico de manera independiente.

Igualmente captó la atención la ausencia de asociación entre una dosis alta de esteroides y el inicio de TB latente, aunque, como se anotó previamente dentro de las limitantes del estudio, el bajo número de pacientes que recibían dosis altas de esteroides, hace que este hallazgo requiera una evaluación más cuidadosa, todavía más si se tiene en cuenta la relación conocida entre uso de esteroides a altas dosis y casos de infecciones oportunistas secundarias a la inmunosupresión.

\section{Conclusiones}

A pesar de todas las limitantes mencionadas, el estudio muestra que aún no existe en este medio una búsqueda lo suficientemente activa de TB latente en los pacientes que reciben terapia biológica, y plantea, además, la necesidad de evaluar cuidadosamente este riesgo, medida que podría cambiar, a futuro, las recomendaciones internacionales en cuanto a la búsqueda de casos e indicación de tratamiento quimioprofiláctico, especialmente si se tiene presente la frecuencia de TB en un país como Colombia. Se abre la puerta, entonces, para nuevos estudios que permitan evaluar de manera acertada la relación entre el uso de terapia biológica y la presencia de TB latente, con el fin último de brindar a los pacientes reumáticos una terapia segura y efectiva.

\section{Bibliografía}

1. Tufariello JM, Chan J, Flynn JL. Latent tuberculosis: mechanisms of host and bacillus that contribute to persistent infection. Lancet Infect Dis. 2003 Sep;3(9):578-90.

2. Cardona P-J, Ruiz-Manzano J. On the nature of Mycobacterium tuberculosis-latent bacilli. Eur Resp J [Internet]. 2004 Dec 24(6):1044-51. [Acceso 10 Feb 2014]; Disponible en: http://www.ncbi.nlm.nih.gov/pubmed/15572551

3. Jasmer R, Nahid P, Hopewell P. Latent tuberculosis infection. New Eng J Med. 2002;347(23):1860-6.

4. Ministerio de Protección Social. Plan Estratégico Colombia Libre de Tuberculosis 2010-2015 Para la Expansión y Fortalecimiento de la Estrategia Alto a la TB. $3^{\text {a }}$. ed. Bogotá: Ministerio de la Protección Social; 2015..

5. Rodríguez Lozano C. Safety of biological therapies: new data from BIOBADASER. Reumatol Clin [Internet]. $2011 \mathrm{Mar}$ 6S3:S1-6 [Acceso 14 Feb 2014]; Disponible en: http://www. ncbi.nlm.nih.gov/pubmed/21794763

6. Gómez-Reino JJ, Carmona L, Valverde VR, Mola EM, Montero MD. Treatment of rheumatoid arthritis with tumor necrosis factor inhibitors may predispose to significant increase in tuberculosis risk: a multicenter active-surveillance report. Arthr Rheum [Internet]. 2003 Aug 48(8):2122-7 [Acceso 3 Feb 2014];.Disponible en: http://www.ncbi.nlm.nih.gov/pub$\mathrm{med} / 12905464$

7. Galloway JB, Hyrich KL, Mercer LK, Dixon WG, Fu B, Ustianowski AP, et al. Anti-TNF therapy is associated with an increased risk of serious infections in patients with rheumatoid arthritis especially in the first 6 months of treatment: updated results from the British Society for Rheumatology Biologics Register with special emphasis on risks in the elderly. Rheumatology (Oxford, England) [Internet]. 2011 Jan ;50(1):12431 [Acceso 11 Feb 2014]; Disponible en: http://www.pubmedcentral.nih.gov/articlerender.fcgi?artid $=3105607 \&$ tool $=$ pmc entrez\&rendertype $=$ abstract

8. Dixon WG, Hyrich KL, Watson KD, Lunt M, Galloway J, Ustianowski A, et al. Drug-specific risk of tuberculosis in patients with rheumatoid arthritis treated with anti-TNF therapy: results from the British Society for Rheumatology Biologics Register (BSRBR). Ann Rheum Dis [Internet]. 2010 Mar 69(3):522-8. [Acceso 3 Feb2014]; Disponible en: http://www. pubmedcentral.nih.gov/articlerender.fcgi?artid=2927681\&too $1=$ pmcentrez\&rendertype $=$ abstract

9. Ja S, Ga W, Christensen R, E TG, Maxwell L, Jk M, et al. Adverse effects of biologics : a network meta-analysis and Cochrane overview (Review ). Cochrane Database of Systematic Reviews. 2011;2(2):1-84.

10. Lalvani A. Diagnosing tuberculosis infection in the 21 st. century: new tools to tackle an old enemy. Chest [Internet]. 2007 Jun 131(6):1898-906. [Acceso 28 Jan 2014];Disponible en: http://www.ncbi.nlm.nih.gov/pubmed/17565023 
11. Schwartzman K. Latent tuberculosis infection: old problem, new priorities. CMAJ. 2002 Mar 19;166(6):759-61.

12. Bresnihan B, Cunnane G. Infection complications associated with the use of biologic agents. Rheum Dis Clin North Am. 2003Feb;29(1):185-202. [Internet]. 2003 Feb;29(1):185-202. Disponible en: http://www.ncbi.nlm.nih.gov/pubmed/12635507

13. Lin PL, Flynn JL. Understanding latent tuberculosis: a moving target. Jf Immunol (Baltimore, Md. : 1950) [Internet]. 2010 Jul 1 185(1):15-22 [Acceso 30 Jan 2014]; Disponible en: http://www.pubmedcentral.nih.gov/articlerender.fcgi?artid=3 $311959 \&$ tool $=$ pmcentrez\&rendertype $=$ abstract

14. Cengiz K, Seker A. Boosted tuberculin skin testing in hemodialysis patients. Am J Infect Contr [Internet]. 2006 Aug 34(6):383-7 [Acceso14 Feb 2014]; Disponible en: http:// www.ncbi.nlm.nih.gov/pubmed/16877108
15. American Thoracic Society. Targeted tuberulin testing and treatment of latent tuberculosis infection. Am J Respi Crit Care Med. 2000;161:s221-47.

16. Centers for disease control and prevention. Morbidity and Mortality Weekly Report World TB Day — March 24, 2010 Decrease in Reported Tuberculosis Cases - United States , 2009. MMWR. 2010;59(10).

17. Keystone K, Papp KA, Wobeser W. Challenges in diagnosing latent tuberculosis infection in patients treated with tumor necrosis factor antagonists. J Rheumatol. 2011;(38):1234-43.

18. Lalvani A, Millington KA. Screening for tuberculosis infection prior to initiation of anti-TNF therapy. Autoimmunity reviews [Internet]. Elsevier B.V.; 2008 Dec 8(2):147-52. [Acceso 23 Jan 2014]; Disponible en: http:/www.pubmedcentral. nih.gov/articlerender.fcgi? artid $=2593775 \&$ tool $=$ pmcentrez\&r endertype $=$ abstract 